\title{
DETERMINANTAL VARIETY AND NORMAL EMBEDDING
}

\author{
KARIN U. KATZ, MIKHAIL G. KATZ, DMITRY KERNER, \\ AND YEVGENY LIOKUMOVICH
}

\begin{abstract}
The space $G L_{n}^{+}$of matrices of positive determinant inherits an extrinsic metric space structure from $\mathbb{R}^{n^{2}}$. On the other hand, taking the infimum of the lengths of all paths connecting a pair of points in $G L_{n}^{+}$gives an intrinsic metric. We prove bilipschitz equivalence between intrinsic and extrinsic metrics on $G L_{n}^{+}$, exploiting the conical structure of the stratification of the space of $n \times n$ matrices by rank.
\end{abstract}

\section{INTRODUCTION}

Consider the group $G L^{+}(n, \mathbb{R})$ of $n \times n$ matrices of positive determinant. It is an open submanifold of the space $\mathbb{R}^{n^{2}}$ of $n \times n$ matrices. Here $G L^{+}(n, \mathbb{R})$ carries two metrics: its extrinsic ambient Euclidean metric with distance function $d_{e x t}$, and the induced intrinsic metric (i.e. least length of path) with distance function $d_{i n t}$. Our main result is the following.

Theorem 1.1. There is a constant $C=C(n)$ such that $d_{\text {int }}<C d_{\text {ext }}$.

Thus the determinantal variety is normally embedded; in other words, the intrinsic and extrinsic metrics are bilipschitz equivalent. We prove Theorem 1.1 first for $n=2,3$ and then for general $n$. The proof uses in an essential way the conical structure of the stratification of the space $\mathbb{R}^{n^{2}}$ of $n \times n$ matrices by rank. Indeed, the extrinsic and intrinsic metrics on a set as simple as $\left\{(x, y): x^{2}-y^{3}>0\right\}$ are clearly inequivalent, due to the fact that the curve $x^{2}-y^{3}=0$ has a cusp.

Bilipschitz equivalence has been studied by a number of authors; see e.g., 1], 2 ], 4], 7]. For a study of the Lipschitz condition in an infinitesimal context see [3, 6].

2010 Mathematics Subject Classification. 53C23, 58A35, 32S60.

Key words and phrases. Determinantal variety, intrinsic metric, bilipschitz equivalence, conical stratification. 


\section{Solution in Dimension 2}

For $2 \times 2$ matrices with coordinates given by the entries $\left(a_{i j}\right)$, the condition $a_{11} a_{22}-a_{12} a_{21}=0$ for the determinantal variety in the space of matrices translates into

$$
\left(a_{11}+a_{22}\right)^{2}-\left(a_{11}-a_{22}\right)^{2}-\left(a_{12}+a_{21}\right)^{2}+\left(a_{12}-a_{21}\right)^{2}=0
$$

or to simplify notation

$$
x^{2}+y^{2}=z^{2}+w^{2} .
$$

Definition 2.1. Let $X_{2}=\left\{A \in \mathbb{R}^{2^{2}}: \operatorname{det}(A)=0\right\}$ denote the determinantal variety.

Lemma 2.2. The intersection $X_{2} \cap S^{3}$ with the unit sphere $S^{3} \subseteq \mathbb{R}^{2^{2}}$ is a flat 2-torus, namely the Clifford torus $T \subseteq S^{3}$.

Indeed, by (2.1) the complement $S^{3} \backslash T$ is a union of two solid tori, consisting respectively of matrices of positive and negative determinant. Note that $X_{2}$ is a linear cone over $T \subseteq \mathbb{R}^{2^{2}}$.

Lemma 2.3. The metrics $d_{\text {int }}$ and $d_{\text {ext }}$ on $T \subseteq \mathbb{R}^{2^{2}}$ are bilipschitz equivalent.

Proof. By rescaling, we can assume that the furthest of the two points is at unit distance from the origin. By compactness, the only problem for a pair of points $p, q$ can arise when the points collide, i.e., $d(p, q)$ tends to 0 . By the smoothness of the Clifford torus, one has $\frac{d_{\text {int }}(p, q)}{d_{e x t}(p, q)} \rightarrow 1$ as $d(p, q) \rightarrow 0$.

Lemma 2.4. Bilipschitz equivalence holds for the intrinsic and the extrinstic metrics on $X_{2}$.

Proof. Let $p, q \in X_{2}$. If the apex $O \in X_{2}$ is one of the points $p, q$ then the intrinsic and the Euclidean distances between them coincide by linearity of the cone.

Thus we can assume that $p \neq O$ and $q \neq O$. To connect a pair of points $p, q$, at different levels in the cone by a path lying in the cone, assume without loss of generality that $p$ is further than $q$ from the apex of the cone. We slide $p$ along the ray toward the apex until it reaches the level of $q$, and then connect it to $q$ by a shortest path contained in that level. The length of the combined path is clearly bilipschitz with the extrinsic distance in the ambient $\mathbb{R}^{2^{2}}$.

Proposition 2.5. The intrinsic and the ambient metrics on the manifold of $2 \times 2$ matrices of positive determinant are bilipschitz equivalent. 
Proof. The closure of $G L^{+}(2, \mathbb{R})$ is a linear cone over the solid torus. Meanwhile, $G L^{+}(2, \mathbb{R})$ itself is the cone without the apex over the $i n$ terior of the solid torus. We consider a straight line path in $\mathbb{R}^{2^{2}}$ connecting a pair of points in $G L^{+}(2, \mathbb{R})$. The length of this path is the extrinsic distance by definition.

The path does not necessarily lie entirely inside $G L^{+}(2, \mathbb{R})$. As the path is straight and the determinantal variety $X_{2}$ is algebraic, the path splits into a finite number of segments satisfying the following:

(1) the interior of each segment lies fully either inside $G L^{+}(2, \mathbb{R})$ or inside the component $G L^{-}(2, \mathbb{R})$;

(2) the endpoints are in $X_{2}$.

Applying Lemma 2.4, we replace every segment that lies in the component $G L^{-}(2, \mathbb{R})$ by an arc that lies in $X_{2}$.

Finally, we push out the arc in $X_{2}$ into $G L^{+}(2, \mathbb{R})$, i.e., replace it by a nearby arc "just inside" $G L^{+}(2, \mathbb{R})$. We will explain the push-out procedure in detail since a similar argument will be used in the general case.

The vertex $O \in X_{2}$ is the unique singular point of the cone, i.e., the complement $X_{2} \backslash\{O\}$ is a smooth manifold. Hence a path in $X_{2}$ disjoint from $O$ can be pushed out infinitesimally into $G L^{+}(2, \mathbb{R})$ by following the normal direction, without significantly affecting its length.

Suppose a path joining $P, Q \in X_{2}$ passes through $O$. Using the linear structure of the cone, the path can be replaced by the shorter path given by the union of the straight line segments $P O \cup O Q \subseteq X_{2}$. Next, $P$ and $Q$ can be replaced by nearby points $P^{\prime}, Q^{\prime} \in G L^{+}(2, \mathbb{R})$. We now form a path $P^{\prime} O \cup O Q^{\prime}$ lying entirely within $G L^{+}(2, \mathbb{R})$ except for the single point $O$.

Let $p \in O P^{\prime}$ be the point of intersection of the segment with a sphere $S(O, \epsilon)$ of small radius $\epsilon>0$ centered at $O$, and similarly for point $q \in O Q^{\prime}$. The intersection $S(O, \epsilon) \cap G L^{+}(2, \mathbb{R})$ is an open solid torus and therefore connected. Hence there is a short path

$$
\gamma \subseteq S(O, \epsilon) \cap G L^{+}(2, \mathbb{R})
$$

joining $p$ to $q$. The resulting path $P^{\prime} p \cup \gamma \cup q Q^{\prime}$ is only slightly longer than the original path joining $P$ and $Q$. This completes the proof of the bilipschitz property for $G L^{+}(2, \mathbb{R})$.

\section{Singularities in Dimension 3}

For $3 \times 3$ matrices, the singular locus of the determinantal variety $X_{3}=\left\{A \in \mathbb{R}^{3^{2}}: \operatorname{det} A=0\right\}$ consists of matrices of rank $\leq 1$. The 
variety $X_{3}$ can be stratified as follows:

$$
X_{3}=X_{3,0} \cup X_{3,1} \cup X_{3,2} .
$$

Here the stratum $X_{3, i}$ consists of matrices of rank $i$. The stratum $X_{3,1}$ lies in the closure of $X_{3,2}$, while $X_{3,0}$ lies in the closure of $X_{3,1}$. Each $X_{3, i}$ is smooth. Here $X_{3,2}$ is of codimension 1 in the space of matrices, while $X_{3,1}$ is of codimension 4 in the space of matrices, and $X_{3,0}$ is a single point.

The closure $\bar{X}_{3,2}$ is a cone on a smooth manifold away from $X_{3,1}$. Thus the only potential obstruction to bilipschitz equivalence is the singularity of $\bar{X}_{3,2}$ along $\bar{X}_{3,1}$, which we now analyze.

Lemma 3.1. The 5-dimensional closure $\bar{X}_{3,1}$ is a linear cone over the smooth compact 4-manifold $\left(S^{2} \times S^{2}\right) /\{ \pm 1\}$.

Proof. Here $S^{2} \times S^{2} /\{ \pm 1\}$ parametrizes the intersection of $X_{3,1}$ with the unit 8-sphere in the space of matrices. The manifold $S^{2} \times S^{2}$ can be parametrized by pairs of unit column vectors $v, w \in S^{2} \subseteq \mathbb{R}^{3}$ producing a rank 1 matrix

$$
v^{t} w \in X_{3,1} .
$$

The element -1 acts simultaneously on both factors of $S^{2} \times S^{2}$ by the antipodal map.

Fix an element $x \in X_{3,1}$. We would like to understand the bilipschitz property for $X_{3}$ in a neighborhood of $x$. Exploiting the action of $G L(3, \mathbb{R}) \times G L(3, \mathbb{R})$ on $X_{3,1}$ by right and left multiplication, we may assume that

$$
x=1 \oplus\left(\begin{array}{ll}
0 & 0 \\
0 & 0
\end{array}\right) .
$$

Definition 3.2. Let

$$
L_{x}=\left\{1 \oplus A: A \in \mathbb{R}^{2^{2}}\right\} \subseteq \mathbb{R}^{3^{2}}
$$

be a transverse slice to $X_{3,1} \subseteq \mathbb{R}^{3^{2}}$ at $x$, so that

$$
L_{x} \cap \bar{X}_{3,2}=\left\{1 \oplus A \in L_{x}: \operatorname{det}(A)=0\right\} .
$$

Note that of course transversality is weaker than orthogonality. The ambient Euclidean metric on $\mathbb{R}^{9}$ plays no role here.

Lemma 3.3. A transverse slice for $X_{3,1} \subseteq \bar{X}_{3,2}$ in local coordinates is a linear cone over a Clifford torus.

Proof. The defining equation (3.1) of the slice is $\operatorname{det}(A)=0$. By Lemma 2.2 this is a cone over a torus. 
Now let $U_{T} \subseteq \mathbb{R}^{5}$ be the unit ball. Choose open sets $U_{N} \subseteq L_{x}$ and $U \subseteq \mathbb{R}^{3^{2}}$, each containing $x$, and a bilipschitz diffeomorphism

$$
\phi: U \rightarrow U_{T} \times U_{N}
$$

such that $\phi\left(U \cap \bar{X}_{3,1}\right)=U_{T} \times\{x\}$ and $\phi\left(U \cap \bar{X}_{3,2}\right)=U_{T} \times\left(U_{N} \cap \bar{X}_{3,2}\right)$. This can be done using the $G L(3, \mathbb{R}) \times G L(3, \mathbb{R})$ action, as explained in [5, p. 138]. Hence Lemmas 3.3 and 2.4 imply that the intrinsic and extrinsic metrics on $U \cap \bar{X}_{3,2}$ are bilipschitz equivalent.

Corollary 3.4. The intrinsic and extrinsic metrics on $\bar{X}_{3,2}=X_{3}$ are bilipschitz equivalent.

This follows from Lemma 3.1 by a compactness argument.

Theorem 3.5. The intrinsic and extrinsic metrics on $G L^{+}(3, \mathbb{R})$ are bilipschitz equivalent.

Proof. The extrinstic distance between a pair of matrices of positive determinant is the length of the straight line path in $\mathbb{R}^{3^{2}}$ joining them. We partition the path into finitely many segments, where the interior of each segment lies entirely in a connected component of $G L(3, \mathbb{R})$ while the endpoints are in $X_{3} \subseteq \mathbb{R}^{3^{2}}$. Then we apply Corollary 3.4 to replace each segment belonging to the component $G L^{-}(3, \mathbb{R})$ by an arc in $X_{3}$. If the arc in $X_{3}$ lies in the smooth part $X_{3,2} \subseteq X_{3}$ then it can be pushed out into $G L^{+}(3, \mathbb{R})$ by a small deformation in the normal direction, as in Section 2 ,

Unlike the case of Proposition 2.5, the determinantal variety is not a cone on a manifold, so that an additional argument is required to push the arc out of $X_{3}$ and into $G L_{3}^{+}$while retaining bilipschitz control.

If an arc in $X_{3}$ joining points $P, Q$ passes through the apex $O \in$ $X_{3}$ then it can be replaced by the union $P O \cup O Q$ and pushed out into $G L^{+}(3, \mathbb{R})$ as in the proof of Proposition 2.5.

Otherwise we exploit the local product structure on $X_{3} \backslash\{O\}$ as in (3.2). A path in $X=X_{3}$ that dips into the singular locus $X_{3,1} \subseteq X$ can be handled as follows. Given a path $\gamma:[0,1] \rightarrow X$, let $a \in[0,1]$ be the least parameter value $t$ such that $\gamma(t)$ is contained in the singular locus $X_{3,1} \subseteq X$, and $b \in[0,1]$ the greatest such value.

Step 1. Consider the restriction of the path $\gamma$ to $[a, b] \subseteq[0,1]$. We replace it by a path that lies entirely in the singular locus $X_{3,1}$. Section 4 proves that $X_{3,1}$ is embedded in $X$ in a bilipschitz fashion. Hence this replacement can be performed in a bilipschitz-controlled way.

Step 2. Once the path $\gamma([a, b])$ is in the singular locus, we exploit a local trivialisation to push it in a constant direction as follows. 
Choose $\delta>0$ sufficiently small to be specified later. Then $\gamma(a-\delta)$ and $\gamma(b+\delta)$ are in the smooth part $X_{3,2} \subseteq X$. The path can easily be shortened to a smooth one, still denoted $\gamma$. Over a sufficiently small neightborhood of the smooth path, we can choose a smooth (and in particular bilipschitz) trivialisation of $X$ over the path $\gamma([a, b])$. Let

$$
I_{0,1}(t)=I_{0,1}=\left(\begin{array}{ll}
1 & 0 \\
0 & 0
\end{array}\right)
$$

be a constant section of the bundle over the path. Relative to the trivialisation of the bundle we can form a new path

$$
\bar{\gamma}_{\epsilon}(t)=\gamma(t)+\epsilon I_{0,1}(t)
$$

which pushes $\gamma([a, b])$ in the constant direction (3.3) for each $t$. By construction, the new path is contained in the nonsingular part $X_{3,2}$ of the determinantal variety, where $\epsilon>0$ is chosen small enough so that the length of the path stays close to the original length of the path in $X_{3,1}$.

Step 3. It remains to check that the path can be patched up with the value of the path $\gamma$ at the parameter values $a-\delta$ and $b+\delta$. Since the determinantal variety in the fiber is a cone over a connected space, the two values can be connected by an arbitrarily short path, provided $\epsilon$ and $\delta$ are chosen small enough.

Step 4. Once the path lies in the nonsingular part $X_{3,2} \subseteq X$ of the determinantal variety, it can be pushed out into $G L^{+}(3, \mathbb{R})$ by following the normal direction as in the case $n=2$.

The higher dimensional case is treated inductively in Section 5 .

\section{The General DETERMinantal VARIETY}

Let $X_{n, k} \subseteq \mathbb{R}^{n^{2}}$ be the stratum of rank $k$ matrices. Thus $X_{n}$ is the closure of $X_{n, n-1}$ and each $X_{n, k}$ is a smooth connected manifold of dimension $n^{2}-(n-k)^{2}$. For each $x \in X_{n, k}$ choose an open set $U_{x} \subseteq \mathbb{R}^{n^{2}}$ containing $x$, a metric ball $U_{x}^{T} \subseteq \mathbb{R}^{n^{2}-(n-k)^{2}}$, an open set $U_{x}^{N} \subseteq \mathbb{R}^{(n-k)^{2}}$ containing 0 , and a bilipschitz diffeomorphism $\phi=\phi_{x}: U_{x} \rightarrow U_{x}^{T} \times U_{x}^{N}$, such that

$$
\phi\left(U_{x} \cap X_{n, k}\right)=U_{x}^{T} \times\{0\}, \quad \phi\left(U_{x} \cap X_{n}\right)=U_{x}^{T} \times\left(U_{x}^{N} \cap X_{n-k}\right) .
$$

See [5, p. 138] for the construction of the diffeomorphism $\phi_{x}$. To ensure the bilipschitz property, it suffices to shrink slightly $U_{x}, U_{x}^{N}$ and $U_{x}^{T}$.

Assume by induction that we have proved the bilipschitz equivalence of the intrinsic and extrinsic metrics on $X_{\ell}$ for $\ell<n$. Generalizing Lemma 3.1 to dimension $n$, we see $\bar{X}_{n, 1}$ is a linear cone over a compact 
smooth submanifold. So, the intrinsic and extrinsic metrics on $\bar{X}_{n, 1}$ are bilipschitz equivalent. For each $x \in X_{n, 1}$, the bilipschitz diffeomorphism $\phi_{x}$ and the induction hypothesis for $X_{n-1}$ show the intrinsic and extrinsic metrics are bilipschitz equivalent on $U_{x} \cap X_{n}$. Then, a compactness argument shows the bilipschitz property holds for a sufficiently small open neighborhood $U_{\epsilon}\left(\bar{X}_{n, 1}\right)$ of $\bar{X}_{n, 1}$ in $X_{n}$. More precisely, $U_{\epsilon}$ is defined to be a cone over an $\epsilon$-neighborhood in the unit sphere.

Next we consider the stratum $X_{n, 2}$. The complement $X_{n, 2} \backslash U_{\epsilon}\left(\bar{X}_{n, 1}\right)$ is a cone on a compact smooth manifold with boundary. For each point $x \in X_{n, 2} \backslash U_{\epsilon}\left(\bar{X}_{n, 1}\right)$, the bilipschitz diffeomorphism $\phi_{x}$ and the induction hypothesis for $X_{n-2}$ show the intrinsic and extrinsic metrics are bilipschitz equivalent on $U_{x} \cap X_{n}$. Arguing by compactness as before, we obtain the bilipschitz property for a sufficiently small neighborhood $U_{\epsilon}\left(\bar{X}_{n, 2}\right)$ of $\bar{X}_{n, 2}$ in $X_{n}$. Here $\epsilon$ may have to be chosen smaller than the one chosen for $X_{n, 1}$.

We proceed in this way until we obtain the bilipschitz property for a neighborhood of $X_{n, n-2}$ in the determinantal variety $X_{n}$. By compactness, the bilipschitz property holds for $X_{n}$ itself.

\section{BILIPSCHITZ PROPERTY FOR THE SET OF MATRICES OF POSITIVE DETERMINANT}

We prove Theorem 1.1 by pushing a path in the determinantal variety out into the component $G L^{+}(n, \mathbb{R})$, and mimicking the proof of Theorem 3.5.

If the path $\gamma$ passes via the apex $O \in X$, it can be replaced by a pair of straight line segments and pushed out into $C$ as in Section 2 .

Otherwise let $k \geq 1$ be the least rank of a matrix $\gamma(t)$ along the path, and let $a_{k} \leq b_{k} \in[0,1]$ be respectively the first and last occurrences of a matrix of rank $k$. As in Section [3, we push the path $\gamma\left(\left[a_{k}, b_{k}\right]\right)$ into $X_{n, k} \subseteq X$, by applying the results of Section 4. We then push it out into $X_{n, k+1}$ by following a constant direction, and patch it up at the endpoints as in Step 3 of the proof of Theorem 3.5.

The new path is in $X_{n, k+1}$. We now choose the corresponding parameter values $a_{k+1}, b_{k+1} \in[0,1]$ and proceed inductively. Thus the path can be pushed out into $X_{n, n-1}$. Finally we follow the normal direction to push the path out into $G L^{+}(n, \mathbb{R})$.

\section{ACKNOWLEDGMENTS}

M. Katz was partially funded by the Israel Science Foundation grant no. $1517 / 12$. D. Kerner was partially supported by the Israel Science Foundation grant 844/14. This paper answers a question posed by Asaf 
Shachar at MO1 and we thank him for posing the question. We are grateful to Yves Cornulier for a helpful comment posted there. We are grateful to Jake Solomon for providing the proof in Section 4 of the general case of the bilipschitz property for the determinantal variety. We thank Jason Starr for a helpful comment posted at MO2 We thank Amitai Yuval for pointing out a gap in an earlier version of the article, and Alik Nabutovsky and Kobi Peterzil for useful suggestions.

\section{REFERENCES}

[1] Birbrair, L.; Fernandes, A.; Lê, D.; Sampaio, J. "Lipschitz regular complex algebraic sets are smooth." Proc. Amer. Math. Soc. 144 (2016), no. 3, 983987.

[2] Birbrair, L.; Mostowski, T. "Normal embeddings of semialgebraic sets." Michigan Math. J. 47 (2000), no. 1, 125-132.

[3] Kanovei, V.; Katz, K.; Katz, M.; Nowik, T. "Small oscillations of the pendulum, Euler's method, and adequality." Quantum Studies: Mathematics and Foundations 3 (2016), no. 3, 231-236.

See http://dx.doi.org/10.1007/s40509-016-0074-x and http://arxiv.org/abs/1604.06663

[4] Katz, K.; Katz, M. "Bi-Lipschitz approximation by finite-dimensional imbeddings." Geom. Dedicata 150 (2011), 131-136.

[5] MacPherson, R.; Procesi, C. "Making conical compactifications wonderful." Selecta Math. (N.S.) 4 (1998), no. 1, 125-139.

[6] Nowik, T., Katz, M. "Differential geometry via infinitesimal displacements." Journal of Logic and Analysis 7:5 (2015), 1-44.

See http://dx.doi.org/10.4115/jla.2015.7.5 and http://arxiv.org/abs/1405.0984

[7] Pedersen, H.; Ruas, M. "Lipschitz Normal Embeddings and Determinantal Singularities." See http://arxiv.org/abs/1607.07746

K. Katz, Department of Mathematics, Bar Ilan University, Ramat GAN 52900 ISRAEL

E-mail address: katzmik@math.biu.ac.il

M. Katz, Department of Mathematics, Bar Ilan University, Ramat GAN 52900 ISRAEL

E-mail address: katzmik@macs.biu.ac.il

D. Kerner, Department of Mathematics, Ben Gurion University of the Negev, Be'ER Sheva 8410501 IsRael

E-mail address: dmitry.kerner@gmail.com

Y. Liokumovich, Imperial College, London

E-mail address: y.liokumovich@imperial.ac.uk

\footnotetext{
${ }^{1}$ See http://mathoverflow.net/questions/222162

${ }^{2}$ See http://mathoverflow.net/questions/230668
} 